\title{
Use of seclusion and restraint, and its relationship to the patient's gender - a retrospective multi-center study from three departments' of acute emergency psychiatry
}

\section{Maria Knutzen}

\author{
Address: Ulleval University Hospital, Psychiatric Division, Kirkeveien 1666, 0407 Oslo, Norway \\ from WPA Thematic Conference. Coercive Treatment in Psychiatry: A Comprehensive Review \\ Dresden, Germany. 6-8 June 2007 \\ Published: 19 December 2007 \\ BMC Psychiatry 2007, 7(SuppI I):P7 doi:10.I I86/I47I-244X-7-SI-P7
}

This abstract is available from: http://www.biomedcentral.com/I47I-244X/7/SI/P7

(c) 2007 Knutzen; licensee BioMed Central Ltd.

\section{Background}

Previous studies show inconsistent findings about gender association with seclusion and restraint. A study from an acute emergency department showed that the number of women secluded and restrained were lower than for the men. But among repeatedly secluded and restrained patients the women were in the majority and they also had more episodes with seclusion and restraint.

\section{Methods}

In an ongoing multi-center study conducted in three acute emergency psychiatry institutions, we retrospectively examine data from seclusion and restraint protocols and data about all the patients admitted over a two-year period. We note how many patients have had episodes with seclusion and restraint. We also note duration/time/ date of each episode and the reason given for using seclusion and restraint and the relationship by gender. Each patient is only counted once in this period, controlling for readmission.

\section{Results}

Preliminary findings from one of the departments replicate findings in the study from the 1994 - 1999 [1], that the total number of men secluded and restrained was higher than that of women, but among repeatedly secluded and restrained patients the women were in the majority. At the congress we will present results from all three departments.

\section{Conclusion}

Our data indicate that the use of seclusion and restraints varies by gender and show the need to study not only the rate of seclusion and restraint, but also the episodes of seclusion and restraint by gender. 